\title{
Article/Artigo
}

\section{Mapping of Chagas disease research: analysis of publications in the period between 1940 and 2009}

\author{
Mapeamento de pesquisa da doença de Chagas: análise da produção de publicações no período \\ entre 1940 e 2009
}

\author{
José Manuel Ramos ${ }^{1}$, Gregorio González-Alcaide ${ }^{2}$,Joaquín Gascón ${ }^{3}$ and Félix Gutiérrez ${ }^{1,4}$
}

\begin{abstract}
Introduction: Publications are often used as a measure of success in research work. Chagas disease occurs in Central and Southern America. However, during the past years, the disease has been occurring outside Latin America due to migration from endemic zones. This article describes a bibliometric review of the literature on Chagas disease research indexed in PubMed during a 70-year period. Methods: Medline was used via the PubMed online service of the U.S. National Library of Medicine from 1940 to 2009. The search strategy was: Chagas disease [MeSH] OR Trypanosoma cruzi [MeSH]. Results: A total of 13,989 references were retrieved. The number of publications increased steadily over time from 1,361 (1940-1969) to 5,430 (20002009) (coefficient of determination for linear fit, $R^{2}=0.910$ ). Eight journals contained $25 \%$ of the Chagas disease literature. Of the publications, $64.2 \%$ came from endemic countries. Brazil was the predominant country (37\%), followed by the United States (17.6\%) and Argentina (14\%). The ranking in production changed when the number of publications was normalized by estimated cases of Chagas disease (Panama and Uruguay), population (Argentina and Uruguay), and gross domestic product (Bolivia and Brazil). Conclusions: Several Latin American countries, where the prevalence of $T$. cruzi infection was not very high, were the main producers of the Chagas disease literature, after adjusting for economic and population indexes. The countries with more estimated cases of Chagas disease produced less research on Chagas disease than some developed countries. Keywords: Chagas disease. American trypanosomiasis. Trypanosoma cruzi. Bibliometry. Scientific production. Mapping.
\end{abstract}

\section{RESUMO}

Introdução: Publicações são frequentemente utilizadas como uma medida de sucesso do trabalho de pesquisa. A doença de Chagas (DCh) ocorre na América Central e do Sul. Porém, durante os últimos anos, a doença tem ocorrido fora da América Latina, devido à migração das zonas endêmicas. Este artigo descreve uma análise bibliométrica da literatura sobre as pesquisas da doença de Chagas indexadas no PubMed, durante um período de 70 anos. Métodos: $\mathrm{O}$ Medline foi usado através do serviço online da US PubMed da National Library of Medicine de 1940 a 2009. A estratégia de busca foi: Chagas disease [MeSH] OR Trypanosoma cruzi [MeSH]. Resultados: Um total de 13.989 referências foi recuperado. O número de publicações aumentou de forma constante com 1.361 (1940-1969) para 5.430 (2000-2009) (coeficiente de determinação para o ajuste linear, $\left.\mathrm{R}^{2}=0,910\right)$. Oito revistas contiveram $25 \%$ da literatura sobre a DCh. Um total de $64,2 \%$ das publicações veio de países endêmicos. O Brasil foi o predominante (37\%), seguido pelos Estados Unidos da América (17,6\%) e Argentina (14\%). O ranking da produção foi alterado, quando o número de publicações foi normalizado por casos estimados da doença de Chagas (Panamá e Uruguai), população (Argentina e Uruguai), e Produto Interno Bruto (Bolívia e Brasil). Conclusões: Vários países da América Latina com uma prevalência não muito elevada de infecção pelo $T$. cruzi foram os principais produtores, após ajuste para os índices econômicos e populacionais. Os países com mais casos estimados da DCh produziu menos pesquisas em doença de Chagas que alguns países desenvolvidos.

Palavras-chaves: Doença de Chagas. Tripanossomíase Americana. Trypanosoma cruzi. Bibliometria. Produção científica. Mapeamento.

1. Unidade de Doenças Infecciosas, Hospital General Universitario de Elche, Alicante, Spain. 2. Departamento de Historia de la Ciencia y Documentación, Facultad de Medicina y Odontología, Universitat de Valencia, Valencia, Spain. 3. Centre de Salut International, Hospital Clinic, Barcelona, Spain. 4. Departamento de Medicina Clinica, Facultad de Medicina, Universidad Miguel Hernández, Alicante, Spain. Address to: Dr. José Manuel Ramos. Unidade de Doenças Infecciosas/Hospital General Universitario de Elche. Camí de l’Almàssera 11, 3203 Elche, Alicante, Spain.

Phone: 34 96661-6754; Fax: 34 96661-6756

e-mail: jramosrincon@yahoo.es

Received in 01/02/2011

Accepted in 01/06/2011

\section{INTRODUCTION}

Chagas disease or American trypanosomiasis is a systemic chronic parasitic infection by Trypanosoma cruzi. Chagas disease occurs throughout Mexico and Central and Southern America. A century ago, Carlos Chagas described the symptoms of the disease, the causative parasitic agent, and the vector. The disease is endemic in 21 countries and continues to present a health threat for an estimated 28 million people, mostly in Latin America ${ }^{1,2}$. Currently, the number of infected persons is estimated at 7.7 million $^{1}$. Chagas disease is a complex zoonosis mainly transmitted by reduviid insects. During the past years, the disease has been occurring outside Latin America, transmitted in a non-vectorial way ${ }^{3,4}$ due to migration from endemic zones ${ }^{5,6}$. Today, the eradication of this disease remains elusive; the clinical manifestations and epidemiological characteristics differ with the diverse endemic zones, and there is a lack of proper treatment. Therefore, the Training and Research in Tropical Diseases (TDR) program and the Pan American Health Organization (PAHO) have identified several key gaps with corresponding research priorities ${ }^{2}$.

Research is important for a country's development and progress ${ }^{7}$. Biomedical research projects usually lead to publications in the serial literature. Original articles allow investigators to present their scientific observations, and the publication of an investigator's project allows the information to be shared by the scientific community. Publications are often used as a measure of success in research work. In recent years, there has been a growing interest in developing scientific indicators capable of facilitating the analysis of the results of research activities ${ }^{7}$.

There are international bibliometric studies in different fields of medicine and/or different diseases ${ }^{8-10}$. There have been recent publications analyzing the research production in parasitology $^{11,12}$, malaria ${ }^{13}$, and tropical medicine ${ }^{14}$, and bibliometric analyses of research published in Tropical Medicine and International Health ${ }^{15}$, Chinese 
Journal of Parasitology and Parasitic Diseases ${ }^{16}$, and Korean Journal of Parasitolog $y^{17}$; of citation patterns in tropical medicine journals ${ }^{18}$, and of anthelmintics ${ }^{19}$. To the best of our knowledge, no quantitative studies analyzing the Chagas disease literature worldwide as well as in specific countries/regions have been carried out in the past. The aim of this study was to investigate the Chagas disease research output over a period of 70 years (1940 to 2009) using PubMed and to explore the extent to which the scientific production parallels the extension of American trypanosomiasis.

\section{METHODS}

The Medline database, accessible free of charge through the PubMed platform, was selected as the most suitable for references on Chagas disease publications. This database is easily accessible and widely used ${ }^{8,20}$. PubMed (http://www.ncbi.nlm.nih.gov/pubmed) was accessed online on 25 May 2010. The subject content analysis of records was conducted according to the structure of the U.S. National Library of Medicine's Medical Subject Headings (MeSH). To retrieve documents, a search was composed with the $\mathrm{MeSH}$ terms or descriptors Chagas disease and Trypanosoma cruzi. The query terms were also searched in the Title and Abstract fields. The period of study was from 1940 to 2009.

The document type was used to refer to the type of article and its funding. The impact factor of a journal and its ranking were copied from the Journal Citation Reports (JCR) 2009 Science Edition ${ }^{21}$. The $\mathrm{MeSH}$ terms were grouped by topic in order to analyze the focal point of the research by considering the following categories: generalized Mesh terms, human/animals, gender, ages, and countries. The number of papers and the percentage of global scientific production for the top $100 \mathrm{MeSH}$ terms were calculated.

Based on geographic, scientific, and economic criteria, the world was divided into six regions: Latin America and the Caribbean, North America, Europe, Asia, Oceania, and Africa. Latin America was considered as an endemic area for American trypanosomiasis; the other regions were considered as non-endemic areas ${ }^{5,22}$. The productivity by country (first participating author) was analyzed by considering the number of papers and the percentage of the world production. In this case, standardized indicators of each country's productivity with respect to the population, the gross domestic product (GDP), and the estimated Chagas disease cases were also provided. To calculate the publication per million inhabitants and the gross domestic product publication per 1,000 million (\$) of the GDP, data were obtained from the World Development Indicators 2009 through the online databases of the World Bank ${ }^{23}$. To calculate the publication by estimated 10,000 Chagas disease cases, indexes were obtained from the T. cruzi seroprevalence estimates of the Pan American Health Organization ${ }^{24}$.

\section{RESULTS}

In the PubMed database, 13,989 references were retrieved for the whole study period, with 1,361 (9.7\%) publications from 1940 to $1969,3,989$ (28.5\%) from 1970-1989, 3,593 (25.7\%) from 1990 to 1999 , and 5,430 (36\%) from 2000 to 2009. Figure 1 shows the numbers of PubMed publications on Chagas disease research during the 70 -year study period by five-year periods. The yearly average increase in publications was $+6.5 \%$ throughout the study period although this percentage was much higher from 1940 to 1969 $(+10.5 \%)$ than from 1970 to $1989(+3.6 \%)$ and from 1990 to 2009
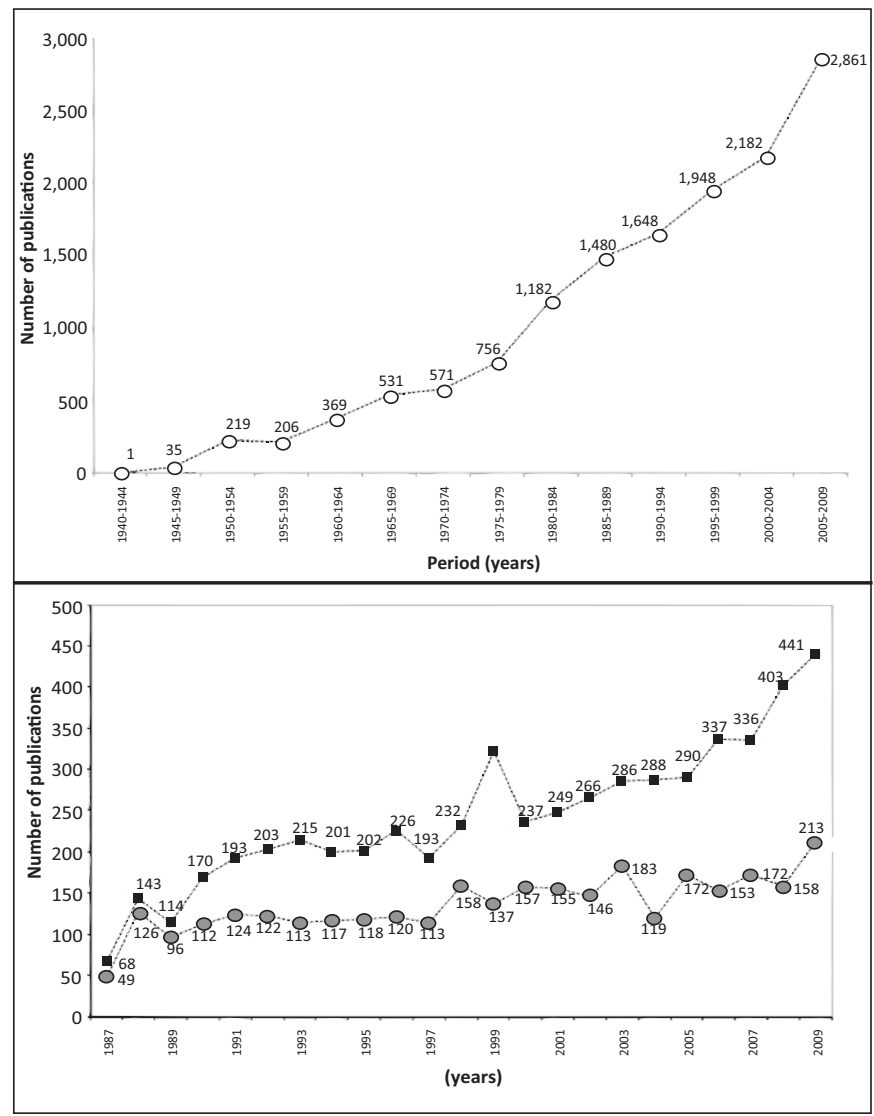

FIGURE 1 - Number of Chagas disease research publications in PubMed (O) between 1940 and 2009 by 5-year periods (Up), and number of Chagas disease research publications in PubMed between 1987 and 2009 by $(\square)$ endemic and (O) non-endemic countries (Down).

$(+4.8 \%)$. After fitting the number of publications over time, a better fit was observed for a straight line (coefficient of determination for linear fit, $\left.R^{2}=0.910\right)$ than for an exponential curve $\left(R^{2}=0.805\right)$.

\section{Language of publication}

The main language was English (76.4\%), followed by Portuguese (11\%), Spanish (9.2\%), French (1.1\%), German (0.6\%), Russian $(0.2 \%)$, Italian $(0.09 \%)$, Japanese $(0.08 \%)$, and others.

\section{Journal of publication}

These articles were published in 1,386 scientific journals. Eight journals contained 25\% of the journal literature on American trypanosomiasis. About half of the literature was concentrated in 35 journals, while the remaining half was scattered over 1,241 journals. Moreover, in 641 journals there was only one paper on Chagas disease published. Table 1 shows a list of the 61 journals with the highest number of papers published during the years 1940-2009, as well as their impact factors for the year 2009, with the journal categories according to JCR classification and language. Twelve of these journals were not included in the JCR because they did not have an impact factor. The other 49 journals were included in at least one of 22 subject categories. These source journals mainly include the fields of Parasitology $(\mathrm{n}=11)$, Immunology $(\mathrm{n}=9)$, Tropical Medicine $(\mathrm{n}=7)$, Microbiology $(\mathrm{n}=6)$, General and Internal Medicine ( $\mathrm{n}=5)$, Public Health $(\mathrm{n}=5)$, and so on.

\section{Analysis of MeSH/subheading words}

The 100 most frequent $\mathrm{MeSH}$ words in the manuscripts about American trypanosomiasis published during the 1940-2009 period are shown in Table 2. The main MeSH after T. cruzi and Chagas disease were Chagas cardiomyopathy (10.2\%), molecular sequence 
TABLE 1 - The 61 journals with the highest number of Chagas disease articles published during the period 1940-2009, their impact factors for the year 2009, the journal category and ranking from the Journal Citation Reports, and the language of publication.

\begin{tabular}{|c|c|c|c|c|c|}
\hline \multirow[t]{2}{*}{ Journal* } & \multicolumn{2}{|c|}{ Articles } & \multirow{2}{*}{$\begin{array}{c}\text { Impact } \\
\text { factor } \\
2009\end{array}$} & \multirow[t]{2}{*}{ Journal category (ranking) } & \multirow[t]{2}{*}{ Language } \\
\hline & n & $\%$ & & & \\
\hline Mem Inst Oswaldo Cruz & 686 & 4.90 & 2.097 & $\begin{array}{l}\text { Parasitology (10 of } 28) \\
\text { Tropical Medicine }(7 \text { of } 17)\end{array}$ & Eng \\
\hline Rev Inst Med Trop São Paulo & 473 & 3.38 & NI & - & $\begin{array}{l}\text { Eng, } \\
\text { Por }\end{array}$ \\
\hline Mol Biochem Parasitol & 462 & 3.30 & 2.939 & $\begin{array}{l}\text { Biochemistry \& Molecular Biology (118 of } 283) \\
\text { Parasitology ( } 7 \text { of } 28)\end{array}$ & Eng \\
\hline Am J Trop Med Hyg & 423 & 3.02 & 2.795 & $\begin{array}{l}\text { Public, Environmental \& Occupational Health } \\
(27 \text { of } 122) \\
\text { Tropical Medicine ( } 3 \text { of } 17)\end{array}$ & Eng \\
\hline Rev Soc Bras Med Trop & 421 & 3.01 & 0.734 & Tropical Medicine (12 of 17$)$ & Mul \\
\hline Exp Parasitol & 399 & 2.85 & 1.773 & Parasitology (13 of 28$)$ & Mul \\
\hline Arq Bras Cardiol & 335 & 2.39 & 1.316 & Cardiac \& Cardiovascular Systems (63 of 95) & Por \\
\hline Trans R Soc Trop Med Hyg & 305 & 2.18 & 2.553 & $\begin{array}{l}\text { Public, Environmental \& Occupational Health } \\
\text { (34 of } 122 \text { ) } \\
\text { Tropical Medicine ( } 4 \text { of } 17 \text { ) }\end{array}$ & Eng \\
\hline Infect Immunity & 265 & 1.89 & 4.205 & $\begin{array}{l}\text { Immunology ( } 27 \text { of } 128) \\
\text { Infectious Diseases (10 of } 57)\end{array}$ & Eng \\
\hline J Parasitol & 264 & 1.89 & 1.195 & Parasitology (19 of 28) & Eng \\
\hline Medicina (Buenos Aires) & 256 & 1.83 & 0.330 & Medicine, General \& Internal (113 of 132) & Mul \\
\hline Acta Trop & 236 & 1.69 & 2.221 & $\begin{array}{l}\text { Parasitology (9 of } 28) \\
\text { Tropical Medicine (6 of } 17)\end{array}$ & Eng \\
\hline Bol Chil Parasitol $^{\mathbf{a}}$ & 201 & 1.44 & $\mathrm{NI}$ & - & Spa \\
\hline Parasitol Res & 188 & 1.34 & 1.721 & Parasitology (14 of 28$)$ & Eng \\
\hline J Immunol & 171 & 1.22 & 5.646 & Immunology (18 of 128) & Eng \\
\hline J Protozool / J Eukaryot Microbiolb & 154 & 1.11 & 2.355 & Microbiology (48 of 94) & Eng \\
\hline Int J Parasitol & 148 & 1.06 & 3.819 & Parasitology (5 of 28$)$ & Eng \\
\hline Rev Bras Pesq Med Biol & 141 & 1.01 & 1.075 & $\begin{array}{l}\text { Biology ( } 44 \text { of } 73) \\
\text { Medicine, Research \& Experimental ( } 74 \text { of 92) }\end{array}$ & Eng \\
\hline J Biol Chem & 139 & 0.99 & 5.328 & Biochemistry \& Molecular Biology (48 of 283) & Eng \\
\hline $\begin{array}{l}\text { Bol Oficina Sanit Panamc/Bull Pan Am Health Organ/Rev } \\
\text { Panam Salud Publica }\end{array}$ & 126 & 0.90 & NI & - & Spa \\
\hline Parasitology & 126 & 0.90 & 1.607 & Parasitology (16 of 28) & Eng \\
\hline Hospital (Rio De Janeiro, Brasil) & 120 & 0.86 & $\mathrm{NI}$ & - & Por \\
\hline Parasitol Today $\mathbf{d} /$ Trends Parasitol & 113 & 0.80 & 4.298 & Parasitology (4 of 28$)$ & Eng \\
\hline Anna Trop Med Parasitol & 110 & 0.79 & 1.368 & $\begin{array}{l}\text { Public, Environmental \& Occupational Health } \\
\text { ( } 72 \text { of } 122) \\
\text { Parasitology (17 of } 28) \\
\text { Tropical Medicine ( } 8 \text { of } 17)\end{array}$ & Eng \\
\hline Rev Bras Malariologia Doenças Trop Publicações Avulsas ${ }^{\mathbf{e}}$ & 107 & 0.76 & NI & 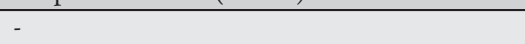 & Mul \\
\hline $\begin{array}{l}\text { Ann Soc Belg Med Tropf/J Trop Med Hyg/Trop Geogr } \\
\text { Med/Trop Med Parasitol/Trop Med \& International } \\
\text { Health }\end{array}$ & 103 & 0.73 & 2.328 & $\begin{array}{l}\text { Public, Environmental \& Occupational Health } \\
\text { ( } 40 \text { of } 122 \text { ) } \\
\text { Tropical Medicine ( } 5 \text { of } 17 \text { ) }\end{array}$ & Mul \\
\hline Int J Cardiol & 102 & 0.73 & 3.469 & Cardiac \& Cardiovascular Systems (24 of 95) & Eng \\
\hline Rev Saude Publica & 95 & 0.68 & NI & - & Por \\
\hline J Med Chem & 79 & 0.56 & 4.802 & Chemistry, Medicinal (3 of 46) & Eng \\
\hline Biochem Biophys Res Commun & 78 & 0.56 & 2.548 & $\begin{array}{l}\text { Biochemistry \& Molecular Biology (146 of } 283) \\
\text { Biophysics ( } 40 \text { of } 74)\end{array}$ & Eng \\
\hline Cad Saude Publica/MS, FIOCRUZ, ENSP & 74 & 0.53 & 0.829 & $\begin{array}{l}\text { Public, Environmental \& Occupational Health } \\
\text { (101 of 122) }\end{array}$ & Por \\
\hline Antimicrob Agents Chemother & 74 & 0.53 & 4.802 & $\begin{array}{l}\text { Microbiology (16 of 94) } \\
\text { Pharmacology \& Pharmacy (24 of 236) }\end{array}$ & Eng \\
\hline J Med Entomol & 73 & 0.52 & 1.921 & $\begin{array}{l}\text { Entomology (10 of } 74) \\
\text { Veterinary Sciences ( } 15 \text { of } 141)\end{array}$ & Eng \\
\hline Rev Bras Biol ${ }^{g}$ & 73 & 0.51 & NI & - & $\begin{array}{l}\text { Eng, } \\
\text { Por }\end{array}$ \\
\hline Rev Med Chil & 72 & 0.51 & 0.487 & Medicine, General \& Internal (106 of 132) & Spa \\
\hline Bioorg Med Chem & 68 & 0.49 & 2.822 & $\begin{array}{l}\text { Biochemistry \& Molecular Biology (125 of } 283) \\
\text { Chemistry, Medicinal ( } 17 \text { of } 46) \\
\text { Chemistry, Organic ( } 17 \text { of } 57)\end{array}$ & Eng \\
\hline Proc Natl Acad Sci U S A & 68 & 0.49 & 9.432 & Multidisciplinary Sciences ( 3 of 48 ) & Eng \\
\hline
\end{tabular}


TABLE 1 - Continue.

\begin{tabular}{|c|c|c|c|c|c|}
\hline \multirow{2}{*}{ Journal* $^{*}$} & \multicolumn{2}{|c|}{ Articles } & \multirow{2}{*}{$\begin{array}{c}\text { Impact } \\
\text { factor } \\
2009\end{array}$} & \multirow{2}{*}{ Journal category (ranking) } & \multirow{2}{*}{ Language } \\
\hline & $\mathbf{n}$ & $\%$ & & & \\
\hline Rev Paulista Med ${ }^{\mathbf{h}}$ & 68 & 0.49 & 0.746 & Medicine, General \& Internal ( 88 of 132$)$ & $\begin{array}{l}\text { Eng, } \\
\text { Por }\end{array}$ \\
\hline Rev Hosp Clin ${ }^{\mathbf{i}}$ Clinics (São Paulo, Brasil) & 66 & 0.47 & NI & Medicine, General \& Internal (47 of 132) & $\begin{array}{l}\text { Por, } \\
\text { Eng }\end{array}$ \\
\hline J Infect Dis & 65 & 0.46 & 5.865 & $\begin{array}{l}\text { Immunology (17 of } 128) \\
\text { Infectious Diseases ( } 4 \text { of } 57) \\
\text { Microbiology (11 of } 94)\end{array}$ & Eng \\
\hline Parasite Immunol & 65 & 0.46 & 2.014 & $\begin{array}{l}\text { Immunology (94 of 128) } \\
\text { Parasitology (12 of } 28)\end{array}$ & Eng \\
\hline J Clin Microbiol & 62 & 0.44 & 4.162 & Microbiology (18 of 94) & Eng \\
\hline Microbes Infect (Institut Pasteur) & 61 & 0.44 & 2.757 & $\begin{array}{l}\text { Immunology ( } 64 \text { of } 128) \\
\text { Microbiology ( } 40 \text { of } 94) \\
\text { Virology (14 of } 30)\end{array}$ & Eng \\
\hline Biochem J & 61 & 0.44 & 5.155 & Biochemistry \& Molecular Biology (50 of 283) & Eng \\
\hline Arch Inst Cardiol $\mathrm{Mex}^{\mathbf{j}} /$ Arch Cardiol Mex & 59 & 0.42 & NI & of & Spa \\
\hline Lancet & 59 & 0.42 & 30.758 & Medicine, General \& Internal (2 of 132) & Eng \\
\hline Immunol Lett & 59 & 0.42 & 2.906 & Immunology (58 of 128) & Mul \\
\hline FEBS Lett & 57 & 0.41 & 3.541 & $\begin{array}{l}\text { Biochemistry \& Molecular Biology (93 of 283) } \\
\text { Biophysics ( } 20 \text { of } 74) \\
\text { Cell Biology ( } 74 \text { of } 161)\end{array}$ & Mul \\
\hline Prensa Medica Argent & 56 & 0.40 & NI & - & Spa \\
\hline Biochim Biophys Actak & 56 & 0.40 & & & Eng \\
\hline Clin Exp Immunol & 54 & 0.39 & 3.009 & Immunology (53 of 128) & Eng \\
\hline Rev Argent Microbiol & 52 & 0.37 & N I & - & Spa \\
\hline Bioorg Med Chem Lett & 51 & 0.36 & 2.650 & $\begin{array}{l}\text { Chemistry, Medicinal (19 of 46) } \\
\text { Chemistry, Organic ( } 22 \text { of } 57)\end{array}$ & Eng \\
\hline J Exp Med & 50 & 0.36 & 14.505 & $\begin{array}{l}\text { Immunology ( } 5 \text { of } 128) \\
\text { Medicine, Research \& Experimental (3 of 92) }\end{array}$ & Eng \\
\hline Am Heart J & 50 & 0.36 & 4.357 & Cardiac \& Cardiovascular Systems (14 of 95) & Eng \\
\hline Rev Bras Med & 48 & 0.34 & $\mathrm{NI}$ & - & Por \\
\hline FEMS Microbiol Lett & 48 & 0.34 & 2.199 & Microbiology (54 of 94) & Mul \\
\hline Arq Neuropsiquiatr & 47 & 0.34 & 0.549 & $\begin{array}{l}\text { Neurosciences ( } 216 \text { of } 220) \\
\text { Psychiatry (100 of } 117)\end{array}$ & Por \\
\hline J Eukaryot Microbiol & 47 & 0.34 & 2.355 & Microbiology (48 of 94) & Eng \\
\hline Acta Cient Venez & 44 & 0.31 & NI & - & Spa \\
\hline Eur J Immunol & 42 & 0.30 & 5.179 & Immunology (20 of 128) & Eng \\
\hline $\begin{array}{l}\text { *The titles of the journals were abbreviated according } \\
\text { Oswaldo Cruz, ENSP: Escola Nacional de Saúde P } \\
\text { ending 2001, merged with Parasitología al Día to fo } \\
\text { Journal of Biology, not indexed in } 2009 \text { JCR Scien } \\
\text { Science Edition. publication year ending 1996, me } \\
\text { Science Edition. publication year ending 1995; me } \\
\text { to form Tropical Medicine \& International Health; } \\
\text { in } 2009 \text { JCR Science Edition. publication year en } \\
\text { by Clinics (São Paulo, Brazil), indexed in } 2009 \text { JCR } \\
\text { Edition. k This journal comprises nine topical secti } \\
\text { BBA: Gene Regulatory Mechanisms; 4) BBA: Ger } \\
\text { 8) BBA: Reviews on Cancer; and 9) BBA: Biomen }\end{array}$ & $\begin{array}{l}\text { ear en } \\
\text { an An } \\
\text { jical } \\
\text { ence E } \\
\text { São Pa } \\
\text { ation y } \\
\text { lly con } \\
\text { lolecu }\end{array}$ & $\begin{array}{l}\text { Consu } \\
\text { Science } \\
\text { not inde } \\
1986 . \\
\text { an Heal } \\
\text { ine and } \\
\text { ne } \\
\text { Medical } \\
\text { anding } 2 \\
\text { red in } 2 \\
\text { d Cell }\end{array}$ & $\begin{array}{l}\text { ttp://www. } \\
\text { lition, Eng: } \\
\text { d in 2009JC } \\
\text { blication ye } \\
\text { Organizatio } \\
\text { ygiene; Tro } \\
\text { ation year en } \\
\text { urnal, index } \\
\text { 0, continue } \\
\text { 9 JCR Scier } \\
\text { logy of Lipi }\end{array}$ & $\begin{array}{l}\text { cbi.nlm.nih.gov/pubmed.MS: Ministério da Saúde, FIOC } \\
\text { nglish, Por: Portuguese, Mul: multilingual, Spa: Spanish. } \\
\text { R Science Edition. publication year ending 2000, contin } \\
\text { ending } 2000 \text {, continued by Trends in Parasitology, inde } \\
\text { to form Revista Panamericana de Salud Pública, not inde } \\
\text { cal and Geographical Medicine, and Tropical Medicine } \\
\text { ling } 1992 \text {, continued by The Journal of Eukaryotic Micro } \\
\text { in } 2009 \text { JCR Science Edition. publication year ending } \\
\text { by Archivos de Cardiología de México, not indexed in } 20 \\
\text { e Edition: 1) BBA: Proteins and Proteomics; 2) BBA: } \\
\text {; 6) BBA: Molecular Basis of Disease; 7) BBA: Molecula }\end{array}$ & $\begin{array}{l}\text { Z: Fundação } \\
\text { lication year } \\
\text { l by Brazilian } \\
\text { in 2009JCR } \\
\text { in 2009JCR } \\
\text { Parasitology } \\
\text { ogy, indexed } \\
\text { 4, continued } \\
\text { JCR Science } \\
\text { nergetics, 3) } \\
\text { ell Research; }\end{array}$ \\
\hline
\end{tabular}

TABLE 2 - The 100 top medical subject headings (MeSH) words in Chagas disease articles published during the period 1940-2009 organized by: generalized MeSH, species MeSH, gender MesH, age MeSH, and country MeSH.

\begin{tabular}{|c|c|c|c|c|c|}
\hline \multirow[b]{2}{*}{ Articles published } & \multicolumn{5}{|c|}{ Generalized MeSH } \\
\hline & $\mathrm{n}$ & $\%$ & & $\mathrm{n}$ & $\%$ \\
\hline Trypanosoma cruzi & 7,383 & 52.8 & Cell Line & 304 & 2.2 \\
\hline Chagas disease & 7,313 & 52.3 & Trypanosoma brucei brucei & 294 & 2.1 \\
\hline Chagas cardiomyopathy & 1,431 & 10.2 & Leishmania & 290 & 2.1 \\
\hline Molecular sequence data & 1,098 & 7.8 & Sensitivity and specificity & 288 & 2.1 \\
\hline Insect vectors & 1,035 & 7.4 & Myocarditis & 287 & 2.1 \\
\hline Antibodies, protozoan & 875 & 6.3 & Glycoproteins & 287 & 2.1 \\
\hline Chronic disease & 871 & 6.2 & Rhodnius & 279 & 2.0 \\
\hline Trypanocidal agents & 847 & 6.1 & Genes, protozoan & 272 & 1.9 \\
\hline Antigens, protozoan & 790 & 5.6 & Neuraminidase & 271 & 1.9 \\
\hline Amino acid sequence & 789 & 5.6 & Disease reservoirs & 269 & 1.9 \\
\hline Protozoan proteins & 743 & 5.3 & Blotting, western & 268 & 1.9 \\
\hline Triatoma & 728 & 5.2 & Electrophoresis, polyacrylamide gel & 266 & 1.9 \\
\hline
\end{tabular}


TABLE 2 - Continue.

\begin{tabular}{|c|c|c|c|c|c|}
\hline \multirow[b]{2}{*}{ Articles published } & \multicolumn{5}{|c|}{ Generalized MeSH } \\
\hline & $\mathrm{n}$ & $\%$ & & $\mathrm{n}$ & $\%$ \\
\hline Trypanosoma & 688 & 4.9 & Insect control & 265 & 1.9 \\
\hline Myocardium & 615 & 4.4 & Lymphocyte activation & 265 & 1.9 \\
\hline Triatominae & 613 & 4.4 & T-Lymphocytes & 259 & 1.9 \\
\hline Base sequence & 547 & 3.9 & Phylogeny & 252 & 1.8 \\
\hline DNA, protozoan & 527 & 3.8 & Antibodies, monoclonal & 251 & 1.8 \\
\hline Electrocardiography & 518 & 3.7 & Sequence alignment & 250 & 1.8 \\
\hline Time factors & 504 & 3.6 & Prevalence & 248 & 1.8 \\
\hline Enzyme-linked immunosorbent assay & 496 & 3.5 & Epitopes & 243 & 1.7 \\
\hline Cells, cultured & 454 & 3.2 & Interferon-gamma & 242 & 1.7 \\
\hline Kinetics & 442 & 3.2 & Molecular weight & 241 & 1.7 \\
\hline Macrophages & 436 & 3.1 & Cysteine endopeptidases & 241 & 1.7 \\
\hline Acute disease & 416 & 3.0 & Serologic tests & 240 & 1.7 \\
\hline Polymerase chain reaction & 408 & 2.9 & RNA, messenger & 237 & 1.7 \\
\hline Species specificity & 387 & 2.8 & Antibodies & 237 & 1.7 \\
\hline Fluorescent antibody technique & 368 & 2.6 & Enzyme inhibitors & 236 & 1.7 \\
\hline Host-parasite interactions & 360 & 2.6 & Culture media & 236 & 1.7 \\
\hline Nitroimidazoles & 352 & 2.5 & Nifurtimox & 235 & 1.7 \\
\hline Trypanosomiasis & 346 & 2.5 & Sequence homology, amino acid & 227 & 1.6 \\
\hline Heart & 346 & 2.5 & Structure-activity relationship & 223 & 1.6 \\
\hline Immunoglobulin G & 340 & 2.4 & Housing & 222 & 1.6 \\
\hline Recombinant proteins & 335 & 2.4 & Leishmaniasis & 217 & 1.6 \\
\hline Parasitemia & 334 & 2.4 & Blood transfusion & 215 & 1.5 \\
\hline Cloning, molecular & 324 & 2.3 & Cross-reactions & 212 & 1.5 \\
\hline Antiprotozoal agents & 322 & 2.3 & DNA & 211 & 1.5 \\
\hline Microscopy, electron & 311 & 2.2 & Isoenzymes & 207 & 1.4 \\
\hline \multirow[t]{3}{*}{ Spleen } & 296 & 2.1 & & & \\
\hline & \multicolumn{5}{|c|}{ Species MeSH } \\
\hline & $\mathrm{n}$ & $\%$ & & $\mathrm{n}$ & $\%$ \\
\hline Animals & 9,710 & 69.4 & Mice, Inbred C57BL & 387 & 2.8 \\
\hline Humans & 6,501 & 46.5 & Disease models, animal & 341 & 2.4 \\
\hline Mice & 2,991 & 21.4 & Dogs & 316 & 2.3 \\
\hline Mice, Inbred Balb C & 677 & 4.8 & Mice, Inbred C3H & 280 & 2.0 \\
\hline \multirow[t]{3}{*}{ Rats } & 649 & 4.6 & Rabbits & 253 & 1.8 \\
\hline & \multicolumn{5}{|c|}{ Gender MeSH } \\
\hline & $\mathrm{n}$ & $\%$ & & $\mathrm{n}$ & $\%$ \\
\hline \multirow[t]{3}{*}{ Male } & 3,143 & 22.5 & Female & 3,098 & 22.1 \\
\hline & \multicolumn{5}{|c|}{ Ages MeSH } \\
\hline & $\mathrm{n}$ & $\%$ & & $\mathrm{n}$ & $\%$ \\
\hline Adult & 1,884 & 13.5 & Child, preschool & 391 & 2.8 \\
\hline Middle-aged & 1,610 & 11.5 & Infant & 333 & 2.4 \\
\hline Adolescent & 835 & 6.0 & Infant, newborn & 259 & 1.9 \\
\hline Aged & 780 & 5.6 & Pregnancy & 257 & 1.8 \\
\hline \multirow[t]{3}{*}{ Child } & 679 & 4.9 & & & \\
\hline & \multicolumn{5}{|c|}{ Countries MeSH } \\
\hline & $\mathrm{n}$ & $\%$ & & $\mathrm{n}$ & $\%$ \\
\hline Brazil & 938 & 6.7 & Mexico & 214 & 1.5 \\
\hline \multirow[t]{3}{*}{ Argentina } & 278 & 2.0 & & & \\
\hline & \multicolumn{5}{|c|}{ Top 30 subheadings on Chagas disease } \\
\hline & $\mathrm{n}$ & $\%$ & & $\mathrm{n}$ & $\%$ \\
\hline Immunology & 3,438 & 24.6 & Drug therapy & 1,038 & 7.4 \\
\hline Parasitology & 3,087 & 22.1 & Etiology & 1,018 & 7.3 \\
\hline Metabolism & 2,865 & 20.5 & Physiopathology & 982 & 7.0 \\
\hline Genetics & 2,279 & 16.3 & Prevention \& control & 917 & 6.6 \\
\hline Physiology & 1,693 & 12.1 & Therapeutic use & 879 & 6.3 \\
\hline Chemistry & 1,690 & 12.1 & Pathogenicity & 694 & 5.0 \\
\hline Analysis & 1,594 & 11.4 & Classification & 676 & 4.8 \\
\hline Epidemiology & 1,522 & 10.9 & Ultrastructure & 606 & 4.3 \\
\hline Diagnosis & 1,498 & 10.7 & Cytology & 564 & 4.0 \\
\hline Enzymology & 1,458 & 10.4 & Veterinary & 316 & 2.3 \\
\hline Pathology & 1,391 & 9.9 & Chemical synthesis & 302 & 2.2 \\
\hline Growth \& development & 1,381 & 9.9 & Mortality & 288 & 2.1 \\
\hline Blood & 1,322 & 9.5 & Therapy & 282 & 2.0 \\
\hline Complications & 1,093 & 7.8 & Microbiology & 259 & 1.9 \\
\hline Transmission & 1,071 & 7.7 & Congenital & 156 & 1.1 \\
\hline
\end{tabular}


data (7.8\%), and insect vector (7.4\%). Table 2 also presents the main subheading or topical qualifiers used for indexing and cataloging the MeSH terms Trypanosoma cruzi and/or American trypanosomiasis and/or Chagas disease. The main subheadings were immunology $(24.6 \%)$, parasitology $(22.1 \%)$, metabolism $(20.5 \%)$, and genetics $(16.3 \%)$.

\section{Document type of publications}

The most common document type was Journal Article, accounting for about $89.3 \%$ of the total $(n=12,489)$. Review and Letter were the second and third most common document types, with 1,057 (7.7\%) and 273 (1.9\%) documents, respectively. Editorial, Congresses and News accounted for the remaining $4 \%$. Only $0.8 \%$ of the documents was subdivided into Controlled Trial $(\mathrm{n}=118), 0.5 \%$ into Evaluation Study $(\mathrm{n}=75)$ and Biography $(\mathrm{n}=73)$, and $0.3 \%$ into Historical Article $(\mathrm{n}=47)$. After 1974 , there was research support in $61.7 \%$ of documents $(7,512 / 12,163)$.

\section{Geographic area and country of publication}

The institutional address of the first author of the publication was present in 8,785 of the 13,989 articles $(62.8 \%)$. The data on the absolute production of articles in each country and world region are presented in Table 3. Latin America and the Caribbean was by far the most productive area in the field of T. cruzi infection, with $64.2 \%$ of all articles having an address in this area. North America ranked second (17.9\%), and Europe ranked third (15.8\%).

Sixty-six countries were represented in the sample (Table 3). Brazil was the predominant country $(37 \%)$. The second was the United States (17.6\%), which belonged to a non-endemic area, and the third was Argentina (14\%). The other main countries from endemic areas are Mexico (3\%), Venezuela (2.9\%), Chile (2.5\%), Colombia (1.4\%), and Uruguay (1.2\%), while those from nonendemic zones were France (4\%), the United Kingdom (3.5\%), Spain (2.9\%), Germany (1.9\%), and Japan (1.3\%).

TABLE 3 - Research output per world region and country based on 8,785 Chagas disease articles published during the study period according to institutional address of the first author.

\begin{tabular}{|c|c|c|c|c|c|c|c|}
\hline Region/country & Number & $\%$ & $\begin{array}{c}\text { Global } \\
\text { ranking }\end{array}$ & Region/country & Number & $\%$ & $\begin{array}{c}\text { Global } \\
\text { ranking }\end{array}$ \\
\hline Latin America \& Caribbean & 5,641 & 64.2 & - & North America & 1,574 & 17.9 & - \\
\hline Endemic countries & - & - & - & USA & 1,523 & 17.3 & 2 \\
\hline Brazil & 3,249 & 37 & 1 & Canada & 54 & 0.6 & 16 \\
\hline Argentina & 1,235 & 14 & 3 & Europe & 1,387 & 15.8 & - \\
\hline Mexico & 263 & 3 & 6 & France & 356 & 4.0 & 4 \\
\hline Venezuela & 257 & 2.9 & 8 & UK & 304 & 3.5 & 5 \\
\hline Chile & 219 & 2.5 & 9 & Spain & 259 & 2.9 & 7 \\
\hline Colombia & 121 & 1.4 & 11 & Germany & 172 & 1.9 & 10 \\
\hline Uruguay & 103 & 1.2 & 13 & Belgium & 82 & 0.9 & 14 \\
\hline Bolivia & 48 & 0.5 & 18 & Sweden & 58 & 0.6 & 15 \\
\hline Panama & 29 & 0.3 & 20 & Switzerland & 54 & 0.6 & 16 \\
\hline Paraguay & 29 & 0.3 & 20 & Italy & 36 & 0.4 & 19 \\
\hline Guatemala & 16 & 0.2 & 22 & Israel & 12 & 0.1 & 25 \\
\hline Peru & 15 & 0.2 & 23 & Russia & 7 & 0.1 & 33 \\
\hline Ecuador & 12 & 0.1 & 25 & Austria & 6 & 0.1 & 34 \\
\hline Costa Rica & 11 & 0.1 & 28 & Slovenia & 6 & 0.1 & 34 \\
\hline Netherlands & 9 & 0.1 & 30 & other countries* & 26 & 0.3 & - \\
\hline Honduras & 9 & 0.1 & 30 & Asia & 148 & 1.7 & - \\
\hline Nicaragua & 4 & 0.0 & 37 & Japan & 117 & 1.3 & 12 \\
\hline El Salvador & 1 & 0.0 & 56 & India & 12 & 0.1 & 25 \\
\hline Belize & 1 & 0.0 & 56 & China & 8 & 0.1 & 32 \\
\hline Suriname & 1 & 0.0 & 56 & other countries ${ }^{* *}$ & 11 & 0.1 & - \\
\hline Non-endemic countries & - & - & - & Oceania & 18 & 0.2 & - \\
\hline Cuba & 10 & 0.1 & 29 & Australia & 14 & 0.1 & 24 \\
\hline Trinidad & 3 & 0.0 & 42 & New Zealand & 4 & 0.0 & 37 \\
\hline Jamaica & 2 & 0.0 & 48 & Africa & 17 & 0.2 & - \\
\hline- & - & - & - & Kenya & 4 & 0.0 & 37 \\
\hline- & - & - & - & South Africa & 4 & 0.0 & 37 \\
\hline- & - & - & - & other countries ${ }^{* * *}$ & 9 & 0.1 & - \\
\hline
\end{tabular}

USA: Unites States of America, UK: United Kingdom, *Ireland ( $n=5)$, Greece $(n=3)$, Portugal $(n=3)$, Norway $(n=2)$, Turkey $(n=4)$, Denmark $(n=2)$, Hungary $(n=2)$, Poland $(n=2)$, Czech Republic $(n=1)$, Slovakia $(n=1)$, Croatia $(n=1) .{ }^{* *} \operatorname{Iran}(n=3)$, Thailand $(n=3)$, Taiwan $(n=2)$, Saudi Arabia $(n=1)$, Myanmar $(n=1)$, Singapore $(n=1) .{ }^{* *}$ Cameroon $(n=3)$, Ethiopia $(n=2)$, Egypt $(n=2)$, Tunisia $(n=1)$, Uganda $(n=1)$. 
Figure 1 shows the number of publications on Chagas disease research in endemic and non-endemic countries. The coefficient of determination for a linear fit was better in endemic countries $\left(\mathrm{R}^{2}=0.860\right)$ than in non-endemic countries $\left(\mathrm{R}^{2}=0.664\right)$.

\section{Publications in endemic countries}

In Table 4, the ranking of countries based on the crude numbers of retrieved articles, and the numbers of publications for the estimated number of Chagas disease cases, inhabitants, and GDP are shown. If we calculated the ratio of the number of American trypanosomiasis publications to the estimated number of Chagas disease cases, Panama, Uruguay, Brazil, and Chile would become the most productive. Normalized by population, the order of prominence would be Argentina, Uruguay, Brazil, and Chile. Normalized by GDP, the leading countries were Bolivia, Brazil, Uruguay, and Argentina.

TABLE 4 - Countries in Latin American and the Caribbean according to total number of publications, publications per estimated Chagas disease (ChD) cases, publications per inhabitants, and documents per gross domestic product (GDP) based on ChD documents published with the institutional address of the first author during the study period.

\begin{tabular}{|c|c|c|c|c|c|c|}
\hline & $\begin{array}{c}\text { ChD } \\
\text { documents } \\
(\text { total } \mathbf{n})\end{array}$ & $\begin{array}{c}\text { Estimated } \\
\text { T. cruzi } \\
\text { prevalence }\end{array}$ & $\begin{array}{c}\text { Estimated } \\
\text { number of } \\
\text { infected }\end{array}$ & $\begin{array}{l}\text { Documents by estimated } \\
10,000 \text { patients } \\
\text { with ChD } \\
\text { (n) }\end{array}$ & $\begin{array}{l}\text { Documents } \\
\text { by million } \\
\text { inhabitants } \\
\text { (n) }\end{array}$ & $\begin{array}{l}\text { Documents by } \\
\text { US\$ 1,000 } \\
\text { million GDP } \\
\text { (n) }\end{array}$ \\
\hline Brazil & 3,249 & 1.02 & $1,919,216$ & 16.93 & 17.27 & 4.23 \\
\hline Argentina & 1,235 & 4.13 & $1,615,051$ & 7.65 & 31.58 & 3.63 \\
\hline Mexico & 263 & 1.03 & $1,073,480$ & 2.45 & 2.52 & 0.39 \\
\hline Venezuela & 222 & 1.16 & 313,560 & 7.08 & 8.21 & 1.51 \\
\hline Chile & 219 & 0.99 & 163,026 & 13.43 & 13.30 & 2.27 \\
\hline Colombia & 121 & 0.96 & 419,563 & 2.88 & 2.77 & 0.99 \\
\hline Uruguay & 103 & 0.66 & 21,875 & 47.08 & 31.08 & 4.12 \\
\hline Bolivia & 48 & 6.75 & 631,383 & 0.76 & 5.13 & 4.48 \\
\hline Panama & 29 & 0.01 & 329 & 882.11 & 8.82 & 1.86 \\
\hline Paraguay & 29 & 2.54 & 152,791 & 1.90 & 4.82 & 3.46 \\
\hline Guatemala & 16 & 1.98 & 257,966 & 0.62 & 1.23 & 0.68 \\
\hline Peru & 15 & 0.69 & 194,414 & 0.77 & 0.53 & 0.21 \\
\hline Ecuador & 12 & 1.74 & 229,733 & 0.52 & 0.91 & 0.56 \\
\hline Costa Rica & 11 & 0.53 & 23,297 & 4.72 & 2.50 & 0.52 \\
\hline Cuba & 10 & 0 & 0 & ND & 0.89 & NA \\
\hline Honduras & 9 & 3.05 & 214,479 & 0.42 & 1.28 & 0.95 \\
\hline Nicaragua & 4 & 1.14 & 62,982 & 0.64 & 0.72 & 0.84 \\
\hline Trinidad & 3 & 0 & 0 & ND & 1.51 & 0.15 \\
\hline Jamaica & 2 & 0 & 0 & ND & 0.75 & 0.20 \\
\hline El Salvador & 1 & 3.37 & 204,953 & 0.05 & 0.16 & 0.07 \\
\hline Belize & 1 & 0.35 & 1,056 & 9.47 & 3.32 & 0.88 \\
\hline Suriname & 1 & 0.18 & 910 & 10.99 & 1.98 & 0.82 \\
\hline
\end{tabular}

NA: not available; ND: not done; ChD: Chagas disease; GDP: gross domestic product.

\section{DISCUSSION}

This study has shown a lineal increase in the number of publications on Chagas disease over the period 1940-2009. The increase in American trypanosomiasis publications seems to be less pronounced than that observed in other diseases and conditions, such as John Cunningham virus or obesity ${ }^{9}$. ${ }^{10}$ This is probably related to the decrease in the prevalence of American trypanosomiasis over the past years and the fact that this disease mostly affects developing countries in Central and South America ${ }^{2}$, which have low expenditures on scientific investigation ${ }^{12}$. The continuous interest in the field and the incorporation of new journals in PubMed may have contributed to this lineal increase.

Although the main language of the Chagas disease research output was English (76.4\%), this language was less predominant in these researches than in other bibliometric studies based on PubMed, where $85-90 \%$ of the documents are in English ${ }^{8,9}$. The other languages were Portuguese (11\%) and Spanish (9.2\%). Chagas disease is endemic to Latin America, which explains the higher prevalence of these languages with respect to other fields recovered from PubMed.

Journal articles were the most commonly retrieved document type $(\approx 90 \%)$, similar to other bibliometric studies ${ }^{8-10}$. Although controlled trials offer the best evidence for the efficacy of medical interventions $s^{25}$, in this study they represented only $0.5 \%$ of the documents, a lower figure than in other fields ${ }^{8,9}$.

Nucleus journals (those with high productivity) usually contain the articles with the highest impact in the area, and thus, subscriptions to such journals by indexing and abstracting services would be justified scientifically ${ }^{10,6}$. Most of the top journals publishing Chagas disease research were from the subject categories Parasitology Immunology and Tropical Medicine. From the top 10 journals, four were from Brazil: Memórias do Instituto Oswaldo Cruz (second quartile in the Parasitology and Tropical Medicine category), Revista do Instituto de Medicina Tropical de São Paulo (not included in Journal Citation Reports), Revista da Sociedade Brasileira de 
Medicina Tropical (third quartile in the Tropical Medicine category), and Arquivos Brasileiros de Cardiologia (third quartile in the Cardiac and Cardiovascular Systems category). The top two non-Latin American journals were Molecular and Biochemical Parasitology (second quartile in the Biochemistry and Molecular Biology, and Parasitology categories) and The American Journal of Tropical Medicine and Hygiene (first quartile in the Tropical Medicine and Public Health category). Twenty-one of the top 61 journals were printed in Latin America (Brazil, 14; Argentina, 3; Chile, 2; and Venezuela, 1), 13 were non-English publications (Portuguese and Spanish), and 12 journals were multilingual publications.

Latin America and the Caribbean was the leading area in scientific production on American trypanosomiasis. This was an expected finding since most people with T. cruzi infection live in this $\operatorname{area}^{2,22,24}$. From non-endemic areas, the highest scientific production came from North America (17.9\%), especially the United States, and Europe (15.8\%). American trypanosomiasis has reached nonendemic zones through migrants from Latin America ${ }^{3-5,22}$. Among countries outside Latin America, the United States led in scientific production on Chagas disease. The field of scientific publications was a very basic theme (immunology, parasitology, and animal model). Other publications came from the Centers for Disease Control and Prevention (Atlanta, Georgia) and the Pan American Health Organization (Regional Office of the World Health Organization localized in Washington). The elevated number of publications from the United States is probably due to the fact that the United States continues to be the most popular country of destination of people infected with T. cruzi, along with the long tradition of U.S. agencies and institutions in implementing research and health programmes, and the network of scientists operating in that country.

The number of Latin American immigrants in Europe has more than doubled from 2001 to $2004^{6,22}$. For example, in Spain the Latin American immigrant population has risen 10-fold from 235,000 in 1998 to 2.45 million in $2009^{27}$. Japan, Australia, and Canada have also received substantial numbers of Latin American immigrants in recent years ${ }^{22}$. The European countries with more Chagas disease scientific researches were France, United Kingdom, Spain, and Germany. France and Spain were more productive than Germany in this field in comparison with other fields ${ }^{8,9}$. This is probably due to the existing network of scientists in France and Spain, with several research teams working on Latin American entomology, parasitology, tropical medicine, and public health. In addition, the movement of people from Latin America to Europe, with an increasing number of them settling there, may play a part. The growing number of cases of American trypanosomiasis in non-endemic countries, the efforts to control congenital and blood-borne routes of transmission ${ }^{3,4,22}$, and the worldwide recognition of Chagas disease as a public health issue would probably translate into an increase in research output in the near future, advancing the search for a better way to diagnose, treat, and prevent the disease.

The analysis of the research production of South America and the Caribbean showed that Brazil was the leading country in publication output on Chagas disease, as has also been described in other biomedical fields ${ }^{8-10}$, followed by Argentina. Brazil, a country with a moderate prevalence of Chagas disease, led in scientific production on Chagas disease research after adjusting for estimated cases of T. cruzi infection behind after Panama and Chile. Argentina, with a higher prevalence of Chagas disease, was the leading country after adjusting for population behind Uruguay. Argentina has a solid reputation in research activity in general, and this is reflected in the investigation on American trypanosomiasis. The other leading countries in publication output on Chagas disease were Mexico and Venezuela, followed by Chile, Colombia, and Uruguay. Panama, with a low prevalence of T. cruzi infection, was the leading country after adjusting for estimated cases of T. cruzi. This was probably due to the fieldwork of a team in Panama city led by the Instituto Conmemorativo Gorgas de Estudios de la Salud. Moreover, Uruguay, with a low prevalence of Chagas disease, was the second leading country after adjusting for estimated cases of American trypanosomiasis. Uruguay has worked hard to control American trypanosomiasis, reducing the incidence of the disease over the last years. This practical experience was reflected in a number of publications initiated by different departments and faculties from the Universidad de la Republica in Montevideo. Chile is another country that, despite having a low number of cases of T. cruzi infection, occupied a leading position in research. Bolivia had the highest prevalence of T. cruzi infection, and its investigators signed as first authors in $0.6 \%$ of the documents. After adjusting for economic parameters, Bolivia emerged as the leading country. Only $0.3 \%$ of the Chagas disease research output was from Paraguay, a country with a high prevalence of T. cruzi infection. In Central America, Honduras and El Salvador had a moderate-to-high prevalence of American trypanosomiasis, but few scientific researches published in journals included in PubMed had investigators from these two countries as first authors.

PubMed and the Science Citation Index were found to be the most suitable databases for searching and retrieving references for bibliometric studies ${ }^{8,20}$. We used the PubMed database because it is easily accessible and widely used ${ }^{8,11,14}$, and the journal index in MedLine has certain criteria for quality ${ }^{20}$. However, the method we used might have had several limitations. For example, the database mainly included journals published in English, and Spanish and Portuguese journals from Latin American countries were less likely to be found on PubMed. Another limitation was that only the address of the first author appeared in the journal articles in PubMed, whereas the address was not recorded at all in Letters and Editorials. Moreover, it was not possible to estimate the quantity of articles that resulted from multinational collaborations. This might have caused some problems in estimating the research productivity in Latin America. Furthermore, finding the addresses in the manuscripts was possible only in publications from the middle of 1987 and not before, and the accuracy of the address of the first author in PubMed was lower than in the Web of Science databases ${ }^{28}$. However, even though the bibliometric methodology used might have presented some limitations, and the results could, in some way, be biased ${ }^{9}$, we believe that this study represents a useful tool for scientists and public health policy makers in planning and organizing research on Chagas disease. We should emphasize that other authors should apply the method used in this study to evaluate research production and that our results may be compared to others in the future. Another limitation of the report related to the calculation of the standardized indicators of each country's productivity with respect to the estimated cases of Chagas disease. We obtained T. cruzi seroprevalence estimates from the Pan American Health Organization report ${ }^{24}$, but the T. cruzi seroprevalence is known to differ depending on the source of the reports. For instance, the nationwide infection rate in Bolivia reported by Schofiel and Dias ${ }^{29}$ was $15.4 \%$, higher than the $6.7 \%$ reported by the Pan American Health Organization. Therefore, the standardized indicators of productivity by 10,000 estimated Chagas disease cases should be interpreted with caution. 
In conclusion, we have found a lineal increase in the number of publications on Chagas disease. Brazil led the scientific production on American trypanosomiasis research. Several countries from Latin America where the prevalence of T. cruzi infection was not very high were the main producers after adjusting for economic and population indexes. Efforts should be made to help those countries with the highest prevalence of Chagas disease, such as Bolivia, Paraguay, Honduras, and El Salvador, to develop a network of scientific research (collaborative platform) with other Latin American countries, and North American or Western European countries to increase the research output. The journal Revista do Instituto de Medicina Tropical de São Paulo and its impact factor were included in the JCR in the year 2010 under the Tropical Medicine subject category (12 of 19).

\section{CONFLICT OF INTEREST}

The authors declare that there is no conflict of interest.

\section{FINANCIAL SUPPORT}

This study was supported by the Fundacion para la Investigacion Hospital General Universitario de Elche (FIBELX08/08). The funders had no role in the study design, data collection and analysis, preparation of the manuscript, or decision to submit the manuscript for publication.

\section{REFERENCES}

1. World Health Organization. Control of Chagas disease. Ginebra: WHO Press; 2002.

2. Organización Mundial de la Salud. Reporte sobre la enfermedad de Chagas. Grupo de trabajo científico 17-20 de abril de 2005 [Internet]. Buenos Aires (AR); 2005 - [cited 2010 May 01] Available from: http,//www.who.int/tdr/ publications/publications/pdf/swg_chagas.pdf.

3. Muñoz J, Portús M, Corachan M, Fumadó V, Gascon J. Congenital Trypanosoma cruzi infection in a non-endemic area. Trans R Soc Trop Med Hyg 2007; 101: 1161-1162.

4. Muñoz J, Coll O, Juncosa T, Vergés M, Del Pino M, Fumado V, et al. Prevalence and vertical transmission of Trypanosoma cruzi infection among pregnant Latin American women attending 2 maternity clinics in Barcelona, Spain. Clin Infect Dis 2009; 48:1736-1740.

5. Schmunis GA, Yadon ZE. Chagas disease, a Latin American health problem becoming a world health problem. Acta Trop 2010; 115:14-21.

6. Pérez de Ayala A, Pérez-Molina JA, Norman F, López-Vélez R. Chagasic cardiomyopathy in immigrants from Latin America to Spain. Emerg Infect Dis 2009; 15:607-608

7. Hefler L, Tempfer C, Kainz C. Geography of biomedical publications in the European Union, 1990-98. Lancet 1999; 353:1856.

8. Ramos JM, Padilla S, Masia M, Gutiérrez F. A bibliometric Analysis of tuberculosis research indexed in PubMed (1997-2006). Int J Tuberc Lung Dis 2008; 12:1461-1468.

9. Vioque J, Ramos JM, Navarrete-Muñoz EM, García-de-la-Hera M. A bibliometric study of scientific literature on obesity research in PubMed (1988-2007). Obes Rev 2010; 11:603-611.

10. Zheng HC, Yan L, Cui L, Guan YF, Takano Y. Mapping the history and current situation of research on John Cunningham virus - a bibliometric analysis. BMC Infect Dis 2009; 9:28.

11. Falagas ME, Papastamataki PA, Bliziotis IA. A bibliometric analysis of research productivity in Parasitology by different world regions during a 9-year period (1995-2003). BMC Infect Dis 2006; 6:56.

12. Falagas ME, Panos G. Implications of findings of bibliometric analyses in parasitology. Trends Parasitol 2007; 23:12-13.
13. Lewison G, Srivastava D. Malaria research, 1980-2004, and the burden of disease. Acta Trop 2008; 106:96-103.

14. Falagas ME, Karavasiou AI, Bliziotis IA. A bibliometric analysis of global trends of research productivity in tropical medicine. Acta Trop 2006; 99:155-159.

15. Glover SW, Bowen SL. Bibliometric analysis of research published in Tropical Medicine and International Health 1996-2003. Trop Med Int Health 2004; 9:1327-1330

16. Lee CS. Bibliometric analysis of the Korean Journal of Parasitology, measured from SCI, PubMed, Scopus, and Synapse databases. Korean J Parasitol 2009; 47: S155-S167.

17. Yang P, Dai J, Gao S, Sheng HF. Bibliometric analysis of the Chinese Journal of Parasitology and Parasitic Diseases in 2006-2008. Zhongguo Ji Sheng Chong Xue Yu Ji Sheng Chong Bing Za Zhi 2009; 27:288-290.

18. Schoonbaert D. Citation patterns in tropical medicine journals. Trop Med Int Health 2004; 9:1142-1150.

19. Zheng Q, Chen Y, Tian LG, Zhou XN. Current situation and developmental trend of anthelmintics by bibliometrics. Zhongguo Ji Sheng Chong Xue Yu Ji Sheng Chong Bing Za Zhi 2009; 27:347-352.

20. Anders ME, Evans DP. Comparison of PubMed and Google Scholar literature searches. Respir Care 2010; 55:578-583.

21. Institute for Scientific Information. Web of Knowledge [Internet]. 2010 - [cited 2010 Ago 1] Available from: http:// www.isinet.com/.

22. Gascon J, Bern C, Pinazo MJ. Chagas disease in Spain, the United States and other non-endemic countries. Acta Trop 2010; 115:22-27.

23. Word Development Indicators [Internet]. - 2009. [cited 2010 Sep 1] Available from: http//databank.worldbank.org/.

24. Organización Panamericana de la Salud. Estimación cuantitativa de la enfermedad de Chagas en las Américas. Montevideo, Uruguay, Organización Panamericana de la Salud. OPS/HDM/CD/425-06. Washington: PAHO Publishing; 2006.

25. Tsay MY, Yang YH. Bibliometric analysis of the literature of randomized controlled trials. J Med Libr Assoc 2005; 93:450-458.

26. López-Illescas C, Moya-Anegón F, Moed HF. The actual citation impact of European oncological research. Eur J Cancer 2008; 44:228-236.

27. Instituto Nacional de Estadística. Avance del Padrón Municipal a 1 de enero 2010 [Internet]. 2010 - [cited 2010 Ago 1] Available at from http://www.ine. es/prensa/np595.pdf/.

28. Clarke A, Gatineau M, Grimaud, O, Royer-Devaux S, Wyn-Roberts N, Le Bis I, et al. A bibliometric overview of public health research in Europe. Eur J Public Health 2007; 17 (suppl I): 43-49.

29. Schofield CJ, Dias JCP. Introduction and historical overview. In: Schofield CJ, Dujardin JP, Jurberg J, editors. Proceedings International Workshop on Population Genetics and Control of Triatominae. Santo Domingo de los Colorados, Ecuador, 24-28 September, 1995. México: Instituto Nacional de Diagnóstico y Referencia Epidemiológicos; 1996. p. 11-16. 